\title{
The Role of Benzylpenicilloyl Epimers in Specific IgE Recognition
}

\author{
Cristobalina Mayorga ${ }^{1,2,3}$, Maria I. Montañez ${ }^{1,3 *}$, Francisco Najera ${ }^{3,4}$, Gador Bogas ${ }^{1,2}$, \\ Tahía D. Fernandez ${ }^{1,5}$, David Rodríguez Gil ${ }^{6}$, Ricardo Palacios ${ }^{6}$, Maria J. Torres ${ }^{1,2,3,7}$, \\ Yolanda Vida ${ }^{3,4 *}$ and Ezequiel Perez-Inestrosa ${ }^{3,4 *}$
}

\begin{abstract}
${ }^{1}$ Allergy Research Group, Instituto de Investigación Biomédica de Málaga-IBIMA, Málaga, Spain, ${ }^{2}$ Allergy Unit, Hospital Regional Universitario de Málaga, Málaga, Spain, ${ }^{3}$ Centro Andaluz de Nanomedicina y Biotecnología-BIONAND, Parque Tecnológico de Andalucía, Málaga, Spain, ${ }^{4}$ Universidad de Málaga-IBIMA Departamento de Química Orgánica, Málaga, Spain, ${ }^{5}$ Universidad de Málaga-IBIMA, Departamento de Biología celular, Genética y Fisiología, Málaga, Spain, ${ }^{6}$ Diater Laboratorios S.A., Madrid, Spain, ${ }^{7}$ Universidad de Málaga-IBIMA, Departamento de Medicina, Málaga, Spain
\end{abstract}

OPEN ACCESS

Edited by:

Alastair George Stewart, The University of Melbourne, Australia

Reviewed by: Concepción González-Bello, Universidad de Santiago de Compostela, Spain Jean-Louis Gueant, Université de Lorraine, France

*Correspondence: Maria I. Montañez maribel.montanez@ibima.eu Yolanda Vida yolvida@uma.es Ezequiel Perez-Inestrosa inestrosa@uma.es

Specialty section: This article was submitted to Translational Pharmacology, a section of the journal Frontiers in Pharmacology

Received: 21 July 2020 Accepted: 13 January 2021 Published: 26 February 2021

Citation:

Mayorga C, Montañez MI, Najera F, Bogas G, Fernandez TD, Gil DR,

Palacios $R$, Torres MJ, Vida $Y$ and Perez-Inestrosa $E$ (2021) The Role of Benzylpenicilloyl Epimers in Specific IgE Recognition.

Front. Pharmacol. 12:585890. doi: 10.3389/fphar.2021.585890
The high prevalence of allergy to $\beta$-lactam antibiotics is a worldwide issue. Accuracy of diagnostic methods is important to prove tolerance or allergy, with skin test considered the best validated in vivo method for diagnosing immediate reactions to $\beta$-lactams. Although drug provocation test is the reference standard, it cannot be performed in highly risk reactions or in those with positive skin tests. For skin tests, the inclusion of major and minor determinants of benzylpenicillin (BP) is recommended. Commercial skin test reagents have changed along time, including as minor determinants benzylpenicillin, benzylpenicilloate (BPO), and benzylpenilloate (PO). Major determinants consists of multivalent conjugates of benzylpenicilloyl coupled through amide bond to a carrier polymer, such as penicilloylpolylysine (PPL) or benzylpenicilloyl-octalysine (BP-OL). The chemical stability of such reagents has influenced the evolution of the composition of the commercial kits, as this requirement is necessary for improving the quality and standardization of the product. In this work, we provide a detailed study of the chemical stability of BP determinants. We observed that those structures suffer from an epimerization process in $\mathrm{C}-5$ at different rates. Butylamine-Benzylpenicilloyl conjugates $(5 R, 6 R)$-Bu-BPO and $(5 S, 6 R)$-Bu-BPO were selected as a simple model for mayor determinant to evaluate the role of the different epimers in the immunoreactivity with sera from penicillin-allergic patients. In vitro immunoassays indicate that any change in the chemical structure of the antigenic determinant of BP significantly affects IgE recognition. The inclusion of stereochemically pure compounds or mixtures may have important implications for both the reproducibility and sensitivity of in vivo and in vitro diagnostic tests.

Keywords: antigenic determinant, diagnostic test, drug allergy, penicillin, specific IgE

\section{INTRODUCTION}

$\beta$-lactam antibiotics (BLs) family is nowadays the first choice for the treatment of a large number of bacterial infections. Its extended use probably explains why they are the drugs most frequently involved in drug hypersensitivity reactions, which have important consequences in terms of safety, durability and effectiveness of treatment. Alternative antibiotics may be less effective, more toxic and expensive, and lead to increased bacterial resistance (Doña et al., 2017). Therefore, a correct diagnosis of drug allergy is very important for an adequate prescription of the drug in order to avoid risks for 


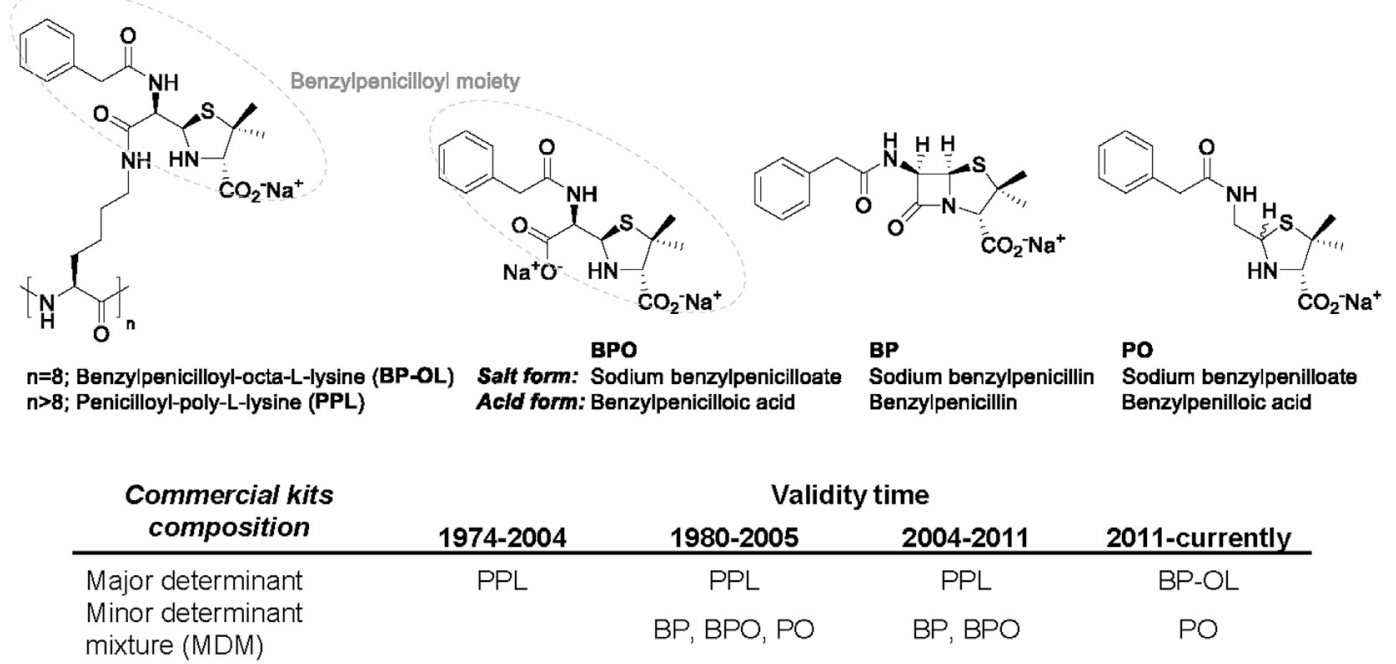

FIGURE 1 | Mayor (BP-OL and PPL) and minor (BPO, BP and PO) determinants of BP traditionally used in skin tests. Evolution of the commercial kits composition over the time.

the patient (Montañez et al., 2015). The first approach for evaluating the patients is a detailed clinical history, which is often very difficult to obtain. After that, the initial choice is frequently skin test (Doña et al., 2019), and in vitro tests which are mainly recommended in patients with dermatological problems, for whom skin test can produce equivocal results, or in high risk patients to reduce the risk of systemic reactions (Mayorga et al., 2016a; Doña et al., 2017). However, in vitro tests are less sensitive than skin test to diagnose penicillin allergy. Although many factors could be involved, one of the most important ones could be the drug or drug metabolite included in the test, in which IgE from patients shows specific recognition (Torres et al., 2010; Mayorga et al., 2016a). Because of patient safety, above described methods are the preferred for diagnosis of drug allergy (Demoly et al., 2014; Doña et al., 2019). However if skin test and in vitro tests are negative, drug provocation tests, also called oral challenge, is the reference standard test required to confirm diagnosis (Torres et al., 2017; Demoly et al., 2014).

The most representative drug model nowadays in the study of immediate (or IgE-mediated) reactions to BLs continues to be Benzylpenicillin (BP) (Levine and Ovary 1961; Batchelor et al., 1965). The main reason is its well-known reactivity based on the nucleophilic attack of free amino groups of proteins to the extremely reactive $\beta$-lactam ring. The opening of the high strain four member ring is an efficient process that leads to the formation of the benzylpenicilloyl determinant. The benzylpenicilloyl amide linked to protein constitutes the reaction product of the $95 \%$ of the penicillin molecules that reacts with proteins under physiological conditions, and it is thus considered the major antigenic determinant of BP. The remaining BP molecules react in a different way, resulting in other structures considered as minor determinants, such as BPO acid form and benzylpenilloic acid (PO) (Martin-Serrano et al., 2016). Many of these structures can be recognized in a different way by IgE from allergic patients, and therefore they are used with diagnostic purposes in in vivo and in vitro tests.

In the case of skin testing, BPO acid, BPO amide forms and PO have been used, showing in some cases higher sensitivity than when using BP itself, probably because the two forms are able to bind better the polyclonal IgE. Extended studies have been carried out since 1977, when the suitability of the major determinant and the different minor determinants was evaluated. In fact, commercial kits have continuously changed over the time. The first commercial kit, in 1974, contained only penicilloyl-poly-L-lysine (PPL) as major determinant. In 1980s, commercial kit included the traditional $\mathrm{BP}$ reagents: $\mathrm{PPL}$ and a minor determinant mixture (MDM), containing $\mathrm{BP}, \mathrm{BPO}$ and $\mathrm{PO}$, Figure 1 . Such kits were removed from the market between 2004 and 2005 (Ariza et al., 2015). In 2004 a different composition mixture was commercialized, including $\mathrm{PPL}$ as major determinant, and only BP and BPO as MDM. In 2011, this formulation was substituted by the more stable benzylpenicilloyl-octa-L-lysine (BP-OL) as major determinant and PO as minor determinant (Fernández et al., 2013; Fernandez et al., 2017). Indeed the diagnosis guidelines from Europe (Maria J. Torres et al., 2003) and North America (Joint Task Force on Practice Parameters et al., 2010) recommended the use of these antigenic determinants of penicillin in skin testing. Nowadays, the commercial determinants in skin test reagents used are BPO-lysine polymer conjugates (BP-OL or PPL, in Europe and United States respectively), together with PO (only in Europe) (Ariza et al., 2015; Martin-Serrano et al., 2016). However, it should be noted that the commercial determinants available depend on the country.

In the case of in vitro tests, immunoassays, which are based on the determination of specific IgE, have been the most widely employed technique. The amide form of BPO covalently bound to PLL is included in solid phase of both commercial and homemade 
immunoassays (Montañez et al., 2011a). In fact, the ImmunoCAP tests available for several penicillins (BP, amoxicillin, ampicillin and penicillin $\mathrm{V}$ ) is based on the penicilloyl-PLL conjugate attached to cellulose solid phase (Fontaine et al., 2007). The homemade RAST (Radio Allergo Sorbent Test) also employs BPO-PLL conjugates attached to a solid phase which, in this case, is a cellulose paper disc. The main reason for the endorse use of such compounds in diagnosis is their high specific recognition by sIgE from penicillin-allergic patients, together with the straightforward reaction of BP with amine nucleophiles and the stability of the penicilloyl determinants formed. This has allowed modifications in homemade RAST assays, in which different carriers and solid phases have been successfully tested (Montañez et al., 2008; Ruiz-Sanchez et al., 2012; Vida et al., 2013; Mayorga et al., 2016b). However, the IgE recognition with BP determinants different from BPO amide structure has not been studied in detail.

Immunoassays are a valuable tool for evaluating the $\operatorname{IgE}$ recognition of the different structures, and in this study we have used them to address the immunological recognition of antigenic determinants and very related chemical structures derived from their storage conditions. In fact, the chemical stability of BP skin test reagents has influenced the evolution of the composition of the commercial kits, as this requirement is necessary for improving the quality and standardization of the product.

Experimental Nuclear Magnetic Resonance (NMR) studies and theorical calculations are suitable methods to get insight into the stability of the determinants and the structural mechanisms concerned. Herein, we report a detailed study of the chemical stability of the BP determinants traditionally used in skin tests, elucidating the chemical process involved, the resulting isomers formed as well as their structural immunoreactivity (or immunological recognition).

\section{MATERIALS AND METHODS}

Standard chemicals were obtained from Aldrich or VWR and used without further purification. Phosphate buffer saline (PBS, $\mathrm{pH} 7.4$ ) was prepared as described elsewhere (Blanca et al., 1992), by dissolving $40 \mathrm{mg}$ of $\mathrm{NaCl}, 1 \mathrm{mg}$ of $\mathrm{KH}_{2} \mathrm{PO}_{4}, 4.5 \mathrm{mg}$ of $\mathrm{Na}_{2} \mathrm{HPO}_{4}$ and $1 \mathrm{mg}$ of $\mathrm{KCl}$ in $5 \mathrm{ml}$ of either $\mathrm{H}_{2} \mathrm{O}$ or $\mathrm{D}_{2} \mathrm{O}$ (99.96\% D, from VWR). Carbonate buffer ( $\mathrm{pH} 10.2)$ was prepared by disolving $14.5 \mathrm{mg}$ of $\mathrm{Na}_{2} \mathrm{CO}_{3}$ and $9.5 \mathrm{mg}$ of $\mathrm{NaHCO}_{3}$ in $5 \mathrm{ml}$ of either $\mathrm{H}_{2} \mathrm{O}$ or $\mathrm{D}_{2} \mathrm{O}$. BP sodium salt, sodium benzylpenicilloate and sodium benzylpenilloate were supplied by DIATER, SA. Benzylpenicilloyl-Butylamine (BuBPO) was prepared as previously described (Sánchez-Sancho et al., 2002). NMR samples were prepared by dissolving $8 \mathrm{mg}$ of the corresponding determinant (BP, BPO, PO or Bu-BPO) in $0.75 \mathrm{ml}$ of the corresponding deuterated solvent and solutions were kept at the indicated temperature until the spectra were recorded. ${ }^{1} \mathrm{H}-\mathrm{NMR}$ spectra were measured in the indicated deuterated solvent on a Bruker Ascend $400 \mathrm{MHz}$ spectrometer. Proton chemical shifts $(\delta)$ are reported with the solvent resonance employed as the internal standard $\left(\mathrm{D}_{2} \mathrm{O} \delta 4.79\right)$.

\section{Computational Studies}

All calculations were performed with the Gaussian 16 package (Frisch et al., 2016). In all simulations, the solvent effect was considered including the polarizable continuum model (PCM) (Tomasi et al., 2005), and water as solvent. The potential energy scans were done for compounds BPO, PO and Bu-BPO at DFT/ B3LYP/6-311G (2 days,p) level of theory using the dihedral angle $\left(\mathrm{H}_{6}-\mathrm{C}_{6}-\mathrm{C}_{5}-\mathrm{H}_{5}\right)$ as variable. The minima obtained in the potential energy scan was used as the initial point for the optimization of their structures at B3LYP/6-311++G (2 days,p) level or theory. The absence of a negative frequency in analytical Hessian calculations confirmed that all the geometries found were minima. To study the intramolecular hydrogens bonds in these molecules, a topological analysis was done using the Bader's atoms in molecules (AIM) theory (Bader 1991), and the Multiwfn 3.6 program (Lu and Chen 2012; Lu 2020).

\section{Selection of Patients}

Patients with a clinical history of an immediate allergic reaction to BP diagnosed following European Academy of Allergy and Clinical Immunology (EAACI) and European Network of Drug Allergy (ENDA) guidelines (Doña et al., 2019; Romano et al., 2020). The studied group was obtained from the Regional University Hospital of Málaga Drug Allergy database, from which we selected eleven cases with a positive skin test and in vitro detection of sIgE to the BP greater than $4.5 \%$, measured by direct RAST. Data from the patients included in the study are displayed in Supplementary Table S1 (ESI).

The study was approved by the institutional review board, and informed consent for all procedures was obtained from all patients.

\section{Skin Test}

Skin prick tests and, if negative, intradermal tests were performed as described (Doña et al., 2019; Romano et al., 2020), using PPL (DAP, Diater, Leganés, Spain) at $1.07 \cdot 10^{-2} \mathrm{M}$, minor determinant mixture (MDM: BP, BPO and PO) at 1.5 M. Since May 2011 DAP composition has changed and includes the major determinant BP-OL at $0.04 \mathrm{mg} / \mathrm{ml}$, equivalent to $8.64 \cdot 10^{-5} \mathrm{M}$ concentration of the benzylpenicilloyl moiety, and the minor determinant (MD) at $0.5 \mathrm{mg} / \mathrm{ml}$, equivalent to $1.5 \cdot 10^{-3} \mathrm{M}$ concentration of PO.

Readings were done after $20 \mathrm{~min}$ and considered positive: 1) In skin prick test, if a wheal larger than $3 \mathrm{~mm}$ surrounded by erythema appeared, with a negative response to the control saline; 2) In intradermal tests, if the increase in diameter of the wheal area marked initially was greater than $3 \mathrm{~mm}$ surrounded by erythema. Positive data expressed as two diameters being one of them the straight line connecting the two most distant points of the wheal and the other the one at $180^{\circ}$ (Brockow et al., 2002).

\section{In vitro Specific IgE Determination}

RAST was done using BP conjugated to PLL functionalizedcellulose discs resulting in BPO-PLL in the solid phase, as described (Antunez et al., 2006; Ariza et al., 2016), and radiolabeled anti-IgE antibody (kindly provided by Thermo Fisher Scientific and radiolabeled in our laboratory) (Martín- 
Serrano et al., 2020). Results were expressed as percentage from a maximum counts and samples were considered positive if the percentage was higher than $2.5 \%$ of label uptake, which was the mean $+2 \mathrm{SD}$ of a negative control group.

\section{Competitive Inhibition Immunoassay}

Solution of inhibitors were prepared as follows. Inhibitor 1: $(5 R, 6 R)-\mathrm{Bu}-\mathrm{BPO}$ was freshly dissolved in PBS; Inhibitor 3: $(5 R, 6 R)-\mathrm{Bu}-\mathrm{BPO}$ in carbonate buffer for 7 days afforded the mixture including $55 \%$ of its epimer, $(5 S, 6 R)-\mathrm{Bu}-\mathrm{BPO}$; Inhibitor 2: a mixture with equivalent volumes of previous samples (inhibitors 1 and 3) resulted in the mixture containing $22.5 \%$ of C-5 epimer. RAST inhibition assay was done as described (Antunez et al., 2006; Ariza et al., 2016), incubating sera from patients and the Bu-BPO determinants (as inhibitors) in three ten-fold decreasing concentrations $(100 \mathrm{mM}-1 \mathrm{mM})$ for $18 \mathrm{~h}$ at room temperature. After this, the BPO-PLL discs were added, and RAST procedure was performed as described above. The results were expressed as percentage inhibition with respect to the serum incubated only with PBS (non-inhibited serum). Comparison of the inhibition capacity of the different inhibitors was performed at $50 \%$ inhibition using the IC50 and statistically analyzed for differences among the distributions of the three inhibitors by Friedman test, Wilcoxon test was used to make comparisons between two pair groups. All assays were performed at room temperature.

\section{RESULTS}

\section{Stability of the Minor Determinants}

NMR Studies were performed to evaluate the stability of the minor determinant reagents used along the last years in solution, in a $7-12 \mathrm{pH}$ range. Solutions of $(3 S, 5 R, 6 R)$-BP resulted in one pure product. In the ${ }^{1} \mathrm{H}-\mathrm{NMR}$ it can be clearly distinguished the signal corresponding to $\mathrm{H}-5, \mathrm{H}-6$ and $\mathrm{H}-3$, bonded to the $\beta$-lactam moiety (Busson and Vanderhaeghe 1976). The compound was completely stable for several days, in both $\mathrm{D}_{2} \mathrm{O}$ and physiological conditions (PBS, $\mathrm{pH} 7.4$ ) (Supplementary Figure S1, ESI).

The obtainment of the BPO determinant from BP is a well described procedure (Munro et al., 1978). The formation of BPO is a spontaneous and efficient process when dissolving BP in aqueous basic media (Pajares et al., 2020). Such reaction could be easily followed by ${ }^{1} \mathrm{H}$-NMR spectroscopy, resulting in a unique compound with a well defined stereochemistry derived from the original BP (Supplementary Figure S2, ESI). (3S,5R,6R)benzylpenicilloate can be easily distinguished from BP by NMR. Signals corresponding to H-5 and H-6 suffer a displacement toward high fields and appear more separated from each other when the $\beta$-lactam ring is opened. Additionally, signal corresponding to $\mathrm{H}-3$ displaces from 4.20 to $3.91 \mathrm{ppm}$. The separation between the signal of both methyl groups of the thiazolidine ring, namely $\mathrm{CH}_{3}(\alpha)$ and $\mathrm{CH}_{3}(\beta)$, increased too.

To evaluate the stability of BPO aqueous solution at different conditions, ${ }^{1} \mathrm{H}-\mathrm{NMR}$ spectra were recorded over time. We observed important changes, indicating the formation of a new product. Signal displacements seem to indicate the formation of a diastereoisomer, in particular the epimer $(3 S, 5 S, 6 R)$ benzylpenicilloate (Ghebre-Sellassie et al., 1984; Haginaka and Wakai 1985). To get more insight into the C-5 epimerization process, the stability of $(3 S, 5 R, 6 R)$-BPO has been tested in aqueous media at different $\mathrm{pH}$ and temperatures. First, a solution of the compound in $\mathrm{PBS} / \mathrm{D}_{2} \mathrm{O}(\mathrm{pH} 7.4)$ at room temperature was monitored over time (Figure 2).

Appearance of new signals was appreciable after $3 \mathrm{~h}$ (Figure 2B). The gap between the chemical shifts of the signals corresponding to the geminal methyl groups increased. Signal corresponding to $\mathrm{H}-5$ displaced to higher field, whereas signal corresponding to H-6 displaced to lower field (from 4.55 to $4.75 \mathrm{ppm}$ ). The coupling constant between $\mathrm{H}-6$ and $\mathrm{H}-5$ also decreased from $5.3 \mathrm{~Hz}$ to $3.8 \mathrm{~Hz}$. All these data confirm the formation of the $(5 S, 6 R)$-BPO epimer. The process evolves until it reaches an equilibrium state after $\sim 30 \mathrm{~h}$, in which approximately $70 \%$ of the $(5 S, 6 R)$-BPO epimer is formed, while a $30 \%$ of the original $(5 R, 6 R)$-BPO isomers remains in solution (Figure 3). To evaluate the effect of $\mathrm{pH}$ in the process, a solution of $(5 R, 6 R)$ - $\mathrm{BPO}$ in $\mathrm{D}_{2} \mathrm{O}$ was prepared and its evolution was monitored. The appearance of the same signal can be clearly observed after 15 h (Supplementary Figure S3, ESI). However, the equilibrium state was not reached until seven days after. The influence of temperature in the process was evaluated. When a solution of $(5 R, 6 R)-\mathrm{BPO}$ in $\mathrm{PBS} / \mathrm{D}_{2} \mathrm{O}(\mathrm{pH} \sim 7.4)$ was monitored cooling at $4^{\circ} \mathrm{C}$, we observed that the process is even slower, taking 7 days to reach a conversion of the $60 \%$ (Figure 3). The same effect was observed when monitoring the process in $\mathrm{D}_{2} \mathrm{O}$ at $4^{\circ} \mathrm{C}$.

Similar studies were made to evaluate the stability in solution of commercial PO, which included $(3 S, 5 R)$ - and $(3 S, 5 S)$ Benzylpenilloate diastereomers. Their solutions at physiological conditions were analyzed both at room $\left(37^{\circ} \mathrm{C}\right)$ and low $\left(4^{\circ} \mathrm{C}\right)$ temperature conditions. ${ }^{1} \mathrm{H}-\mathrm{NMR}$ spectra of the freshly prepared solutions show two products (Supplementary Figure S4, ESI), corresponding to 50:50 mixture of the PO diastereomers in C-5. The solution is stable, observing the same mixture (50:50 ratio) without any degradation product, after $48 \mathrm{~h}$ at room temperature, or 7 days at $4^{\circ} \mathrm{C}$, at least. Similar behavior is observed when the solution is prepared using $\mathrm{D}_{2} \mathrm{O}$ as solvent (Supplementary Figure S5, ESI).

Table 1 shows the conversion rates for the epimerization of $(5 R, 6 R)-\mathrm{BPO}$ and the equimolar mixture of $(5 R)$-PO and $(5 S)$ PO. Differences can be clearly observed between both compounds. For BPO, it is also noticeable the influence of the $\mathrm{pH}$ and temperature on the process, which is accelerated in basic media and high temperature.

\section{Stability of the Major Determinants}

As previously exposed, the mayor determinants currently used in skin tests are BPO-lysine polymer conjugates (BP-OL or PPL). In order to simplify the study, a low molecular weight amine functionalized compound was used instead of amine functionalized polymers to generate the amide-BPO derivatives. We chose benzylpenicilloyl-butylamine (Bu-BPO) 


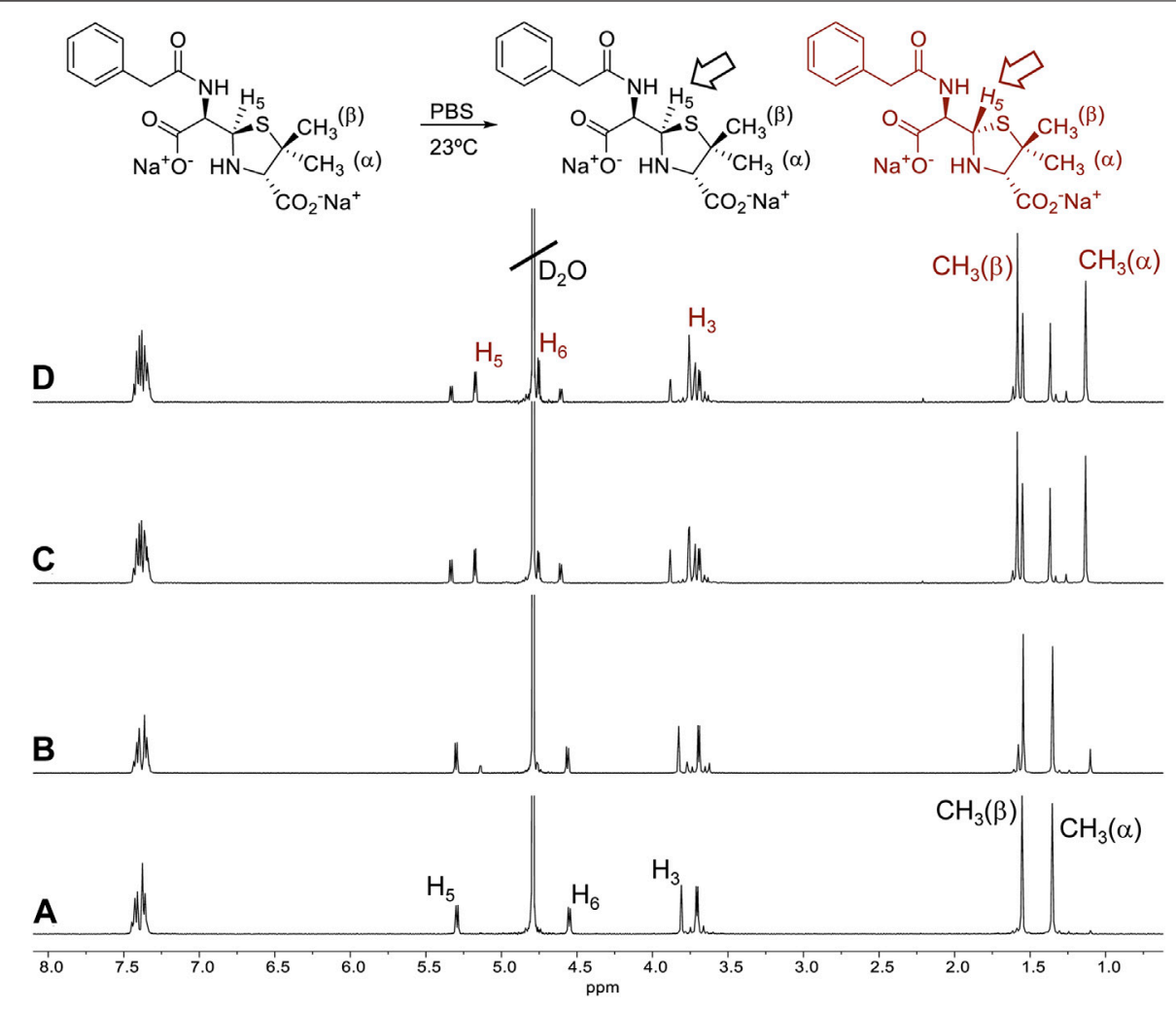

FIGURE $\left.2\right|^{1} \mathrm{H}-\mathrm{NMR}$ spectra of a solution of $\mathrm{BPO}$ in $\mathrm{PBS} / \mathrm{D}_{2} \mathrm{O}, \mathrm{pD} \sim 7.4$ at $23^{\circ} \mathrm{C}$, (A) freshly prepared and after (B) $3 \mathrm{~h}$, (C) $24 \mathrm{~h}$ and (D) $48 \mathrm{~h}$.
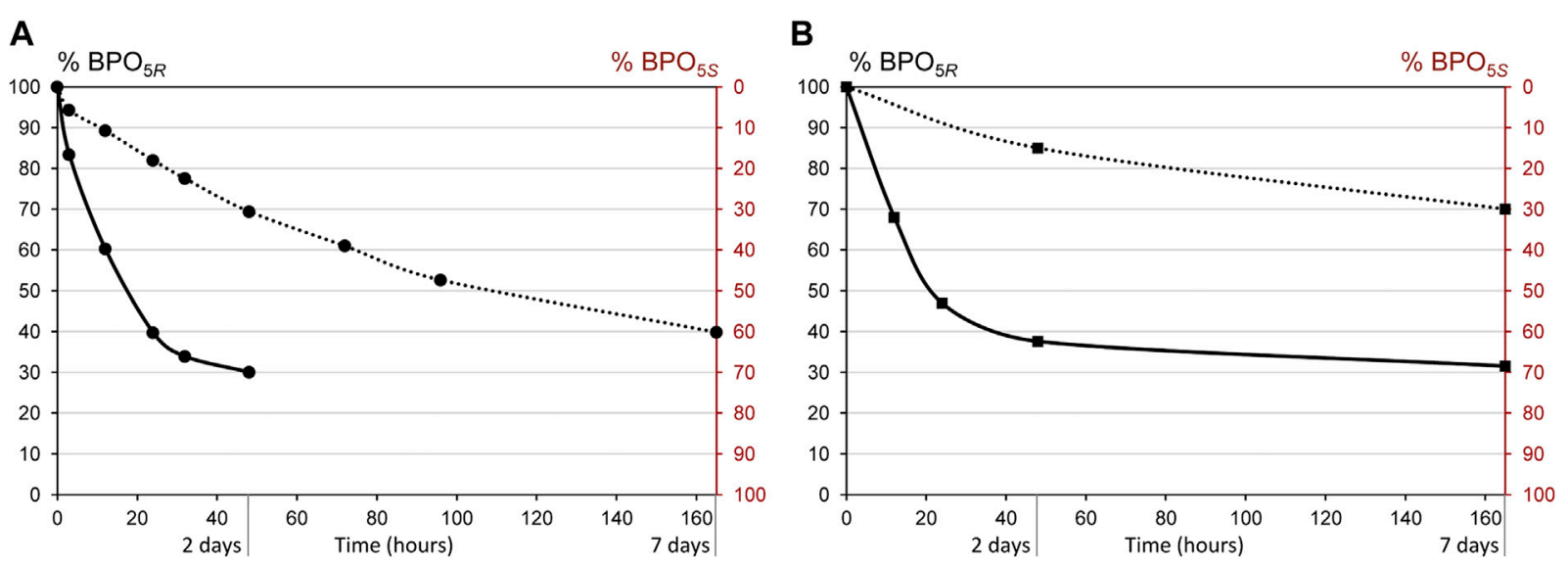

FIGURE 3 | Stability of $\mathrm{BPO}$ in (A) PBS/ $\mathrm{D}_{2} \mathrm{O}, \mathrm{pD} 7.4$ and (B) $\mathrm{D}_{2} \mathrm{O}, \mathrm{pD}=6$, at $23^{\circ} \mathrm{C}$ (solid line) and $4^{\circ} \mathrm{C}$ (dotted line).

as model monomer compound. The reaction between butylamine and $\mathrm{BP}$ in aqueous media yielded the product corresponding to the aminolysis of the $\beta$-lactam ring (Bu-BPO) instantaneously (Sánchez-Sancho et al., 2002: Montañez et al., 2011b). The stability of Bu-BPO has been tested in similar conditions to those studied for minor determinants.

Solutions of Bu-BPO in aqueous media and PBS resulted perfectly stable at room temperature for 7 days
(Supplementary Figures S6, S7, ESI), since signals corresponding to $\mathrm{Bu}-\mathrm{BPO}$ in ${ }^{1} \mathrm{H}-\mathrm{NMR}$ spectra do not change in these conditions. To evaluate the stability of the determinant in basic media, solutions of Bu-BPO in carbonate buffer $(\mathrm{pH} 10.2)$ at room temperature were evaluated. The formation of the $(5 S, 6 R)$-Bu-BPO epimer can be observed in the NMR spectrum after $48 \mathrm{~h}$ (Supplementary Figure S8, ESI). The process evolves 
TABLE 1 | Conversion rates of the minor determinants epimerization process.

\begin{tabular}{|c|c|c|c|c|}
\hline \multirow[b]{2}{*}{ Time/T ( $\left.{ }^{\circ} \mathbf{C}\right)$} & \multicolumn{2}{|c|}{$\mathrm{PBS} / \mathrm{D}_{2} \mathrm{O}(\mathrm{pH} \sim 7.4)$} & \multicolumn{2}{|c|}{$\mathrm{D}_{2} \mathrm{O}(\mathrm{pH} \sim 6)$} \\
\hline & $23 \pm 2^{\circ} \mathrm{C}$ & $4 \pm 1^{\circ} \mathrm{C}$ & $23 \pm 2^{\circ} \mathrm{C}$ & $4 \pm 1^{\circ} \mathrm{C}$ \\
\hline \multicolumn{5}{|c|}{$\%(5 R, 6 R)$-BPO remaining in solution } \\
\hline $\mathrm{Oh}$ & 100 & 100 & 100 & 100 \\
\hline $24 \mathrm{~h}$ & 40 & 82 & 47 & \\
\hline $48 \mathrm{~h}$ & 30 & 69 & 38 & 85 \\
\hline 7 days & & 40 & 30 & 70 \\
\hline \multicolumn{5}{|c|}{$\%(5 R)$-PO remaining in solution } \\
\hline $\mathrm{Oh}$ & 50 & 50 & 50 & 50 \\
\hline $48 \mathrm{~h}$ & 50 & & 50 & 50 \\
\hline 7 days & & 50 & & \\
\hline
\end{tabular}

TABLE 2 | Conversion rates of Bu-BPO epimerization process.

Time $\quad \mathrm{D}_{2} \mathrm{O}(\mathrm{pH} \sim 6) \quad \mathrm{PBS} / \mathrm{D}_{2} \mathrm{O}(\mathrm{pH} \sim 7.4) \quad \begin{gathered}\text { Carbonate } \\ \text { buffer } / \mathrm{D}_{2} \mathrm{O}(\mathrm{pH}=10.2)\end{gathered}$

$\%(5 R, 6 R)$-Bu-BPO remaining in solution

$\begin{array}{llll}0 \mathrm{~h} & 100 & 100 & 100 \\ 48 \mathrm{~h} & 100 & 100 & 70 \\ 7 \text { days } & 100 & 100 & 45\end{array}$

until approximately $55 \%$ of the $(5 S, 6 R)$ - Bu-BPO epimer is formed, while a $45 \%$ of the original $(5 R, 6 R)$ - $\mathrm{Bu}$-BPO isomers remain in solution after 7 days (Table 2).

\section{Immunological Studies}

The ability of IgE in sera from BP-allergic patients to recognize BP determinants bearing different stereochemistry was studied by RAST inhibition. This assay consists in competitive serum IgE recognition between the solid phase (PLL-BPO conjugate attached to cellulose) and the different inhibitors (Bu-BPO 1) and its mixture with different percentage of its epimer at C-5 (2 $(22.5 \%)$ and $3,(55 \%))$ at different concentrations in the fluid phase.

BPO-Bu compound was selected because it presents the higher stability in aqueous solution at neutral $\mathrm{pH}$, which permits controlling the precise chemical structures of inhibitors. Moreover, inhibitor 3, consisting of a mixture containing a $55 \%$ of its epimer in C-5 (45:55, 5R6R:5S6R) obtained when reached equilibrium in basic aqueous media, was evaluated. In addition, inhibitor 2, a mixture containing midpoint of above concentrations of Bu-BPO and its epimer in C-5 (77.5:22.5, 5R6R: $5 S 6 R$ ) was also included.

The immunological evaluation of $\mathrm{Bu}-\mathrm{BPO}$, and its mixtures with its epimer at C-5, by RAST inhibition (Figure 4A) showed, in most cases, a concentration dependent inhibition of BPspecific sera, with inhibitors following similar patterns in each serum independently of the BP-specific IgE levels, with the best recognition obtained with the inhibitor 1 , with higher content of (5R,6R)-Bu-BPO.

In vitro IgE recognition is normally considered meaningful and positive when the inhibition percentage is higher than $50 \%$. In general, at the maximum concentration of the determinants $(100 \mathrm{mM})$ there was a positive inhibition of $100 \%$ of sera for inhibitors one and two and of $81.8 \%$ for the inhitor 3 . This inhibition dropped at $1 \mathrm{mM}$ concentration, being positive in three out of 11 cases (27\%) for inhitor one and one out 11 cases (9\%) for inhibitors 2 and 3, which contain the epimer.

In order to evaluate the role of the epimer in sIgE recognition from allergic patients, we calculated the half-maximal inhibitory concentration (IC50) for each inhibitor represented in Figure 4B. The median and interquartilic ranges of IC50 were 3.32 (IQ: 0.96-7.7), 7.19 (IQ:2.14-9.57) and 15.11 (IQ: 7.64-329.3) for inhibitors 1, two and three respectively, indicanting a decrease of recognition with the increase of the epimer concentration. Statistical comparisons by Friedman test showed significant differences for the three inhibitors $(p=0.0002)$. The concentration to get $50 \%$ of inhibition was significantly lower for the inhibitor 1 , which contains only $(5 R, 6 R)-\mathrm{Bu}-\mathrm{BPO}$ compared with the inhibitors 2 and 3, which contain $22.5(p=$ $0.037)$ and $55 \%(p=0.004)$ of C-5 epimer, respectively. Moreover, we found significant differences in the IC50 between the inhibitors 2 and 3, which contain 22.5 and $55 \%$ of the epimer, respectively $(p=0.002)$ (Figure $4 B$ ).

\section{DISCUSSION}

BP consumption has decreased over the three last decades, mainly substituted by amoxicillin (with or without clavulanic acid) and cephalosporins. However, skin test to BP determinants is still recomended in the BL allergy work up diagnosis (Doña et al., 2017). Different compositions have been used over time, including as minor determinants: $\mathrm{BP}$ (active principle), BPO (the hydrolysis product), and $\mathrm{PO}$ (the decarboxilated $\mathrm{BPO}$ ); and as major determinants conjugates of lysine polymers: PPL and BP-OL. The change in compositions can be explained by stability issues (which must overcome quality and standardization requirements of the product in different countries) and diagnostic results. In fact, a recent study concluded that skin testing with BP can induce falsepositive results in patients with a history of $\mathrm{BL}$ allergy, and that the addition of BP does not increase the skin test sensitivity obtained with classic BP determinants (Lacombe-Barrios et al., 2016). Moreover, false positive results issues have also been reported in commercial in vitro tests, ImmunoCAP, in patients with suspected IgE-mediated hypersensitivity to penicillins and a positive penicillin ImmunoCAP due to the presence of $\operatorname{IgE}$ antibodies to phenylethylamine. This is a common allergenic structure that shares structural fragments (benzyl group) of the penicillin determinants and inappropriately, is potentially present in the ImmunoCAP test (Johansson et al., 2013). In addition, experts from the United States have reported that ImmunoCAP to quantify sIgE to BP shows suboptimal sensitivity and low concordance with in vivo tests, probably because this immunoassay only identifies IgE to the major determinant (Macy et al., 2010).

Although BPO is no longer the most relevant hapten in immediate reactions to penicillins, major and minor determinants of BP continue to play a key role in drug allergy diagnosis (Fernández et al., 2013). These determinants consist of the precise chemical structures involved in the specific $\operatorname{IgE}$ molecular recognition. Therefore the stability of these molecules is an important factor for diagnosis evaluation, as a small change in 
A
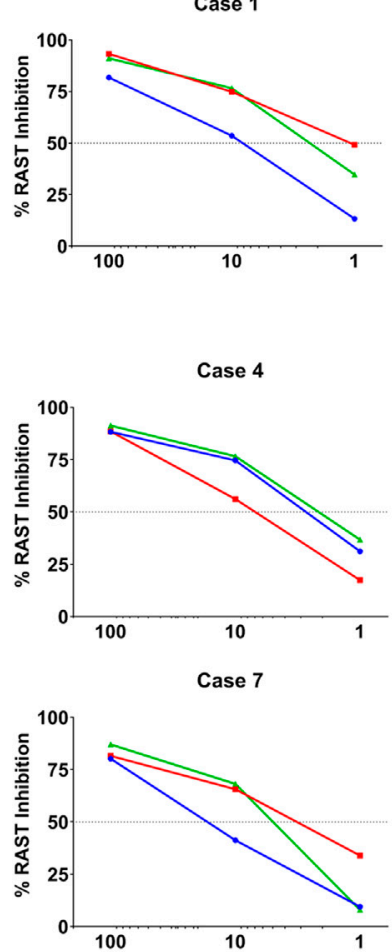

RAST $>20 \%$

Case 2

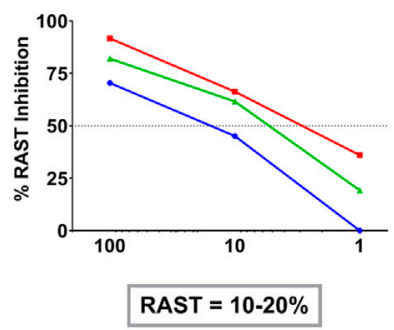

Case 5

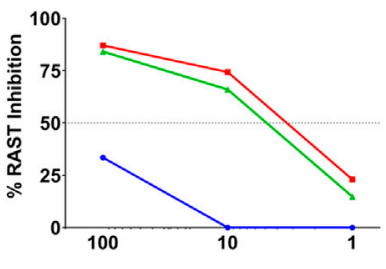

Case 8

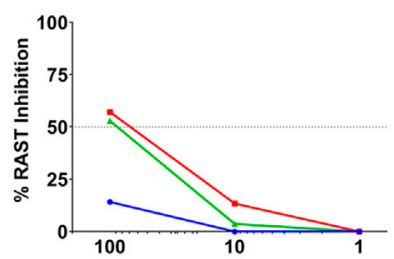

\section{RAST $<10 \%$}

Case 10

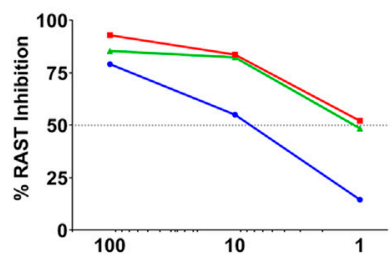

Inhibitor concentration (mM)

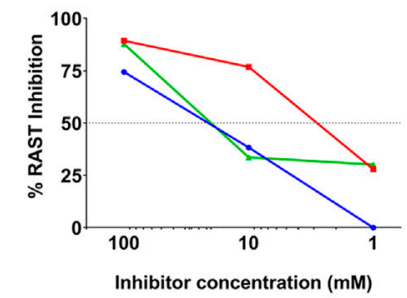

Case 3

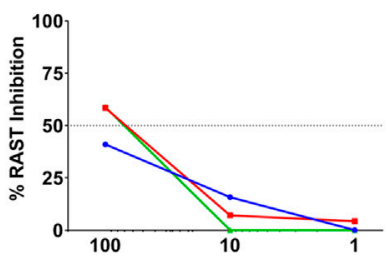

Case 6

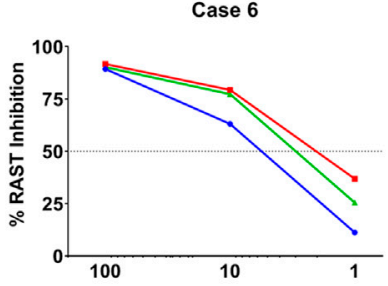

B
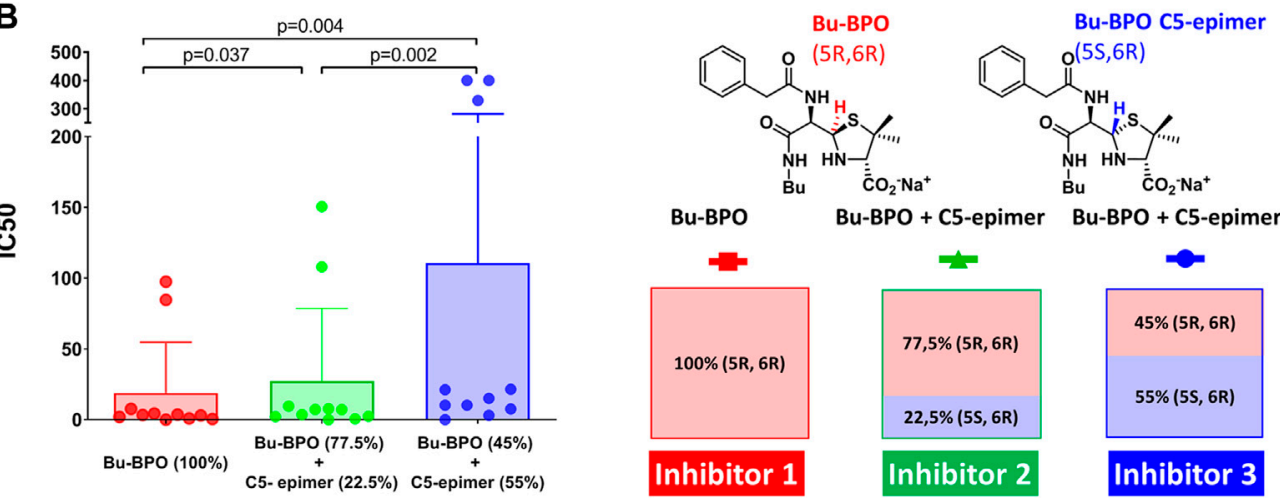

FIGURE 4 | RAST inhibition assays. (A) Results obtained with eleven serum samples from patients allergic to penicillins with different BP-slgE levels. The Bu-BPO and the mixtures including its epimer at C-5, at different proportion, were used as fluid phase inhibitors at three concentrations. Dots lines indicate $50 \%$ of inhibition in which slgE recognition is considered. (B) IC50 for the three kinds of inhibitors (Bu-BPO and the two mixtures with its epimer in C-5) from the eleven sera included. Friedman test indicated statistically significant differences between the distributions of the three inhibitors $(p=0.0002)$. Wilcoxon test was used to make comparisons between two pair groups. 


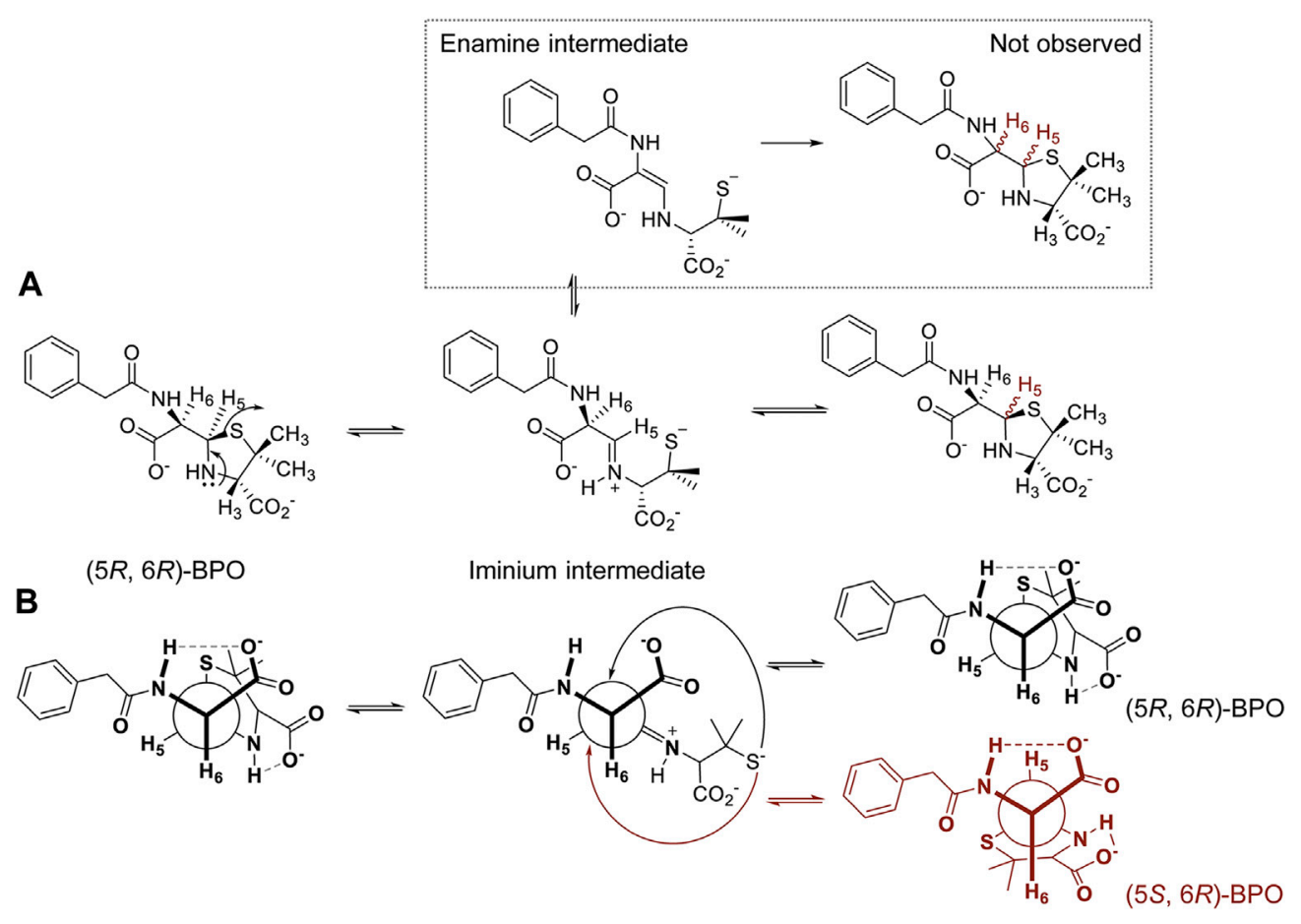

FIGURE 5 | Epimerization process of $(5 R, 6 R)$-BPO. (A) Proposed mechanism through an iminium intermediate and (B) same mechanism represented by the C6C5 bond Newman projection.

the structure can affect the immunological recognition. Moreover, the stability of the determinants can also affect the reproducibility of the tests, which is a crucial point in diagnosis. In that sense, the stability of the determinants used has been evaluated in aqueous solutions in different $\mathrm{pH}$ conditions, ranging from $\mathrm{pH} 7$ to 10.2. $\mathrm{BP}$ resulted stable enough in 6-7.2 $\mathrm{pH}$ range, however, by increasing basicity the $\beta$-lactam ring opens, resulting in the formation of BPO (Munro et al., 1978).

According to previous studies, BP degradation products, formed in vitro when the drug is longer in aqueous solution, are the sensitization agents rather than the BP molecule itself. Although authors did not identify these degradation products, we could assume that BPO and its epimer are involved (Neftel et al., 1984; Neftel et al., 1982).

In the present study, $(5 R, 6 R)$-BPO initially formed resulted not completely stable in solution. While maintaining the carbon skeleton of the structure, the stereochemistry of one of the three stereocenters is altered (Ghebre-Sellassie et al., 1984; Haginaka and Wakai 1985). Although the formation of different degradation products had been previously proposed (Davis et al., 1991), epimerization rate is considerably greater and the formation of other products is almost negligible. The compound evolved reaching an equilibrium in which $70 \%$ of the $(5 S, 6 R)$ BPO epimer is formed. The mechanism involved has been proposed through both the formation of an emanime (Davis and Page 1985) or the formation of an iminum intermediate (Ghebre-Sellassie et al., 1984; Branch et al., 1987; Llinás et al., 2001). However, the enamine pathway has been proposed to occur at $\mathrm{pH}$ above 12 (Davis et al., 1991), and has not been considered in this study, since the epimerization of C-6 is not observed experimentally (Figure 5).

In 7-7.4 $\mathrm{pH}$ range, the formation of an iminium intermediate promotes the opening of the thiazolidine ring. This implies breaking the covalent bond between $\mathrm{C}-5$ and the sulfur atom in position 1, and therefore the free rotation between C-3, C-2 and $\mathrm{S}-1$. This is a reversible process in which the ring closure through the intramolecular nucleophilic attach from the sulfur atom to the iminium C-5 can occur from the two different faces of the iminium, forming thus the two diastereoisomers observed. This closing process could be expected to occur faster than the conformational change, however, as the ring closure is a 5-endotrig process, which is assumed to be unfavorable (Baldwin 1976), it may allow sufficient time for a conformational change. This process is described to be faster at higher $\mathrm{pH}$, being almost immediate in strong basic media (Davis et al., 1991). We also observed a strong influence of temperature, reaching the equilibrium in $48 \mathrm{~h}$ in $\mathrm{PBS} / \mathrm{D}_{2} \mathrm{O}$ at room temperature but taking 7 days at $4^{\circ} \mathrm{C}$. More than one week is necessary to reach the equilibrium when the solution is in $\mathrm{D}_{2} \mathrm{O}$. Experimentally, no hydrolysis of the iminium intermediate or intramolecular reaction between C-3 carboxylate and C-5 has been observed.

$\mathrm{PO}$ is the current available minor determinant reagent for skin test. Its formation may involve C-5 epimerization process, since the two epimers $(50: 50 \%)$ can be observed in freshly prepared solutions. These are very stable compounds in aqueous solutions, as they contain $50 \%$ of the product retaining original stereochemistry of BP for the studied time (seven days). 


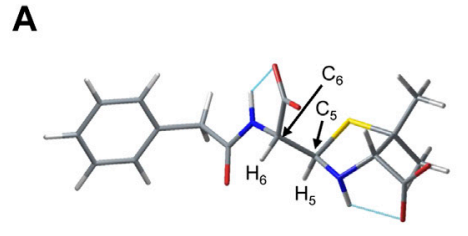

Energy $(\mathrm{kcal} / \mathrm{mol}) \quad-944460.66$ Relative energy $(\mathrm{kcal} / \mathrm{mol}) \quad 86524.2$
B

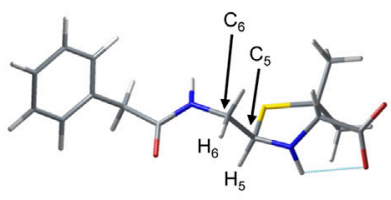

$-826377.73$ 204607.13
C

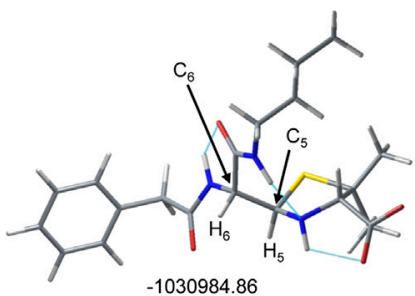

FIGURE 6 | DFT Simulation at PCM $\left(\mathrm{H}_{2} \mathrm{O}\right) / \mathrm{B} 3 \mathrm{LYP} / 6-311++\left(2\right.$ days,p)//PCM $\left(\mathrm{H}_{2} \mathrm{O}\right) / \mathrm{B} 3 \mathrm{LYP} / 6-311(2$ days,p) of $\mathbf{( A )}(5 R, 6 R)-\mathrm{BPO}, \mathbf{( B )}(5 R)-\mathrm{PO}$ and $\mathbf{( C )}(5 R, 6 R)-\mathrm{Bu}-$ $\mathrm{BPO}$ and their relative energies.

$\mathrm{Bu}-\mathrm{BPO}$ was used as model compound to evaluate the stability of the major determinats reagents for skin test. Bu-BPO resulted completely stable in aqueous solutions and PBS at room temperature. Epimerization was oserved when increasing basic conditions to $\mathrm{pH}=10.2$, where the $\mathrm{C}-5$ epimerization ocurrs, but with a slower rate that in the case of BPO. Seven days were necessary to observe the formation of $55 \%$ of the $(5 S, 6 R)-\mathrm{Bu}-\mathrm{BPO}$ epimer. This is consistent with previous results described in the bibliography in which the esterification of the carboxy group reduced considerably the rate of spontaneous thiazolidine ring opening (Davis et al., 1991).

To deepen the stability of the different compounds, DFT calculations were made. In Figure 6 the most stable conformations of $(5 R, 6 R)-\mathrm{BPO},(5 R)-\mathrm{PO}$ and $(5 R, 6 R)-\mathrm{Bu}-\mathrm{BPO}$ are shown.

We can observe the formation of intramolecular hydrogen bonds that could contribute to the stabilization of the molecules. In all cases, a hydrogen bond is observed between the carboxylic group in position three and the hydrogen bonded to N-4 of the thiazolidine ring (cyane lines in Figure 6). In the case of BPO, the formation of another hydrogen bond is observed between the carboxylic acid and the amide moiety bonded to C-6. This extra interaction is not possible in the case of $\mathrm{PO}$, since this carboxylic group is not present in the $\mathrm{PO}$ structure, and this could be translated into a more efficient epimerization process. In contrast, in the case of Bu-BPO, we can also observe the formation of a third intramolecular hydrogen bond, formed between the amide moiety of the butyl substituent and the N-4 of the thiazolidine ring. In this case, the formation of an extra hydrogen bond could make the epimerization process for Bu-BPO less successful. Electronic effects can also contribute to this effect, since the formation of the third hydrogen bong involves that N-4 of the thiazolidine ring does not have its electron pair available for the iminium formation, minimizing thus the epimerization rate. Althougth the formation of the hydrogen bonds is the main difference observed between $\mathrm{PO}, \mathrm{BPO}$ and $\mathrm{Bu}-\mathrm{BPO}$ molecules, we can not exclude other effects when molecules are in saline solutions. It is noteworthy that the DFT calculations using a polarizable continuum model (PCM) results in a negligible relative energy difference between the epimers $((5 R)-\mathrm{PO} /(5 S)$ $\mathrm{PO} ;(5 R, 6 R)-\mathrm{BPO} /(5 S, 6 R)-\mathrm{BPO}$ and $(5 R, 6 R)-\mathrm{Bu}-\mathrm{BPO} /(5 S, 6 R)-$ $\mathrm{Bu}-\mathrm{BPO}$ ), see ESI), so the driving force for the epimerization process is not clear.
To gain insight into how the relative spatial arrangement of a simple carbon (C-5) in the determinant structure can affect the interaction with the immune system, we selected a determinant model that allows the in vitro evaluation of the product retaining original BP stereochemistry, as well as constant proportions of its epimer. Running RAST inhibition experiments requires the inhibitors to be in neutral $\mathrm{pH}$ aqueous solution for $18 \mathrm{~h}$. Since $\mathrm{BPO}$ is not stable at this conditions, due to epimerization; and PO is already supplied as mixture of epimers (50:50), Bu-BPO was selected as model determinant that allows evaluating both epimers as controling the content of epimers is possible. Therefore, we studied the correlation between Bu-BPO chemical structures and their recognition by sIgE from patients allergic to BP. From the analysis of data, we have observed that, as a general trend, sIgE recognizes $\mathrm{Bu}-\mathrm{BPO}$ determinants with a lower degree of recognition with increasing proportion content of the C-5 epimer. The way in which the stereochemistry of $\mathrm{C}-5$ of Bu-BPO affects IgE recognition is independent on the patient and the sIgE levels. This higher IgE specificity to $(5 R, 6 R)$-Bu- $\mathrm{BPO}$, which preserves the initial stereochemistry of $\mathrm{BP}$, is better visualized with IC50 values. Significant lower concentrations to get the same $50 \%$ of inhibition were needed for the inhibitor one compared with the inhibitors two and three to get the same $50 \%$ of inhibition. These findings indicate, as a general rule, that both epimers of $\mathrm{Bu}$ $\mathrm{BPO}$ determinants are specifically recognized by sera from BPallergic patients, although the tridimensional conformation of C5 seems to refine the extent of recognition in a high percentage of cases.

These stability and sIgE recognition data could be somewhat extrapolated about their implications into current skin reagents of $\mathrm{BP}$, and their diagnostic implications, as follows: 1) the major determinant, $\mathrm{BP}-\mathrm{OL}$, is stable enough in solution to perform the in vivo assay, as no epimerization of this benzylpencilloyl amide form (Bu-BPO) occurs at neutral $\mathrm{pH} ; 2)$ the minor determinant, $\mathrm{PO}$, includes both epimers at 50:50 proportion, which is stable and constant to perform the in vivo assay; 3 ) regarding the $\mathrm{BPO}$ minor determinant $((5 R, 6 R)$-BPO (acid)), although it would contain higher amount of the one with sterochemistry better recognized in vitro (inhibitor 1), since it epimerizes in neutral $\mathrm{pH}$ solution, the reagent present in the skin test would always be a mixture of compounds in different proportions, impairing its sensitivity and impeding its reproducibility. 


\section{CONCLUSION}

The chemical stability of BP skin test reagents has influenced the evolution of the composition of the commercial available kits available, as this requirement is necessary for improving the quality and standardization of the product. Althought the epimerization of $(5 R, 6 R)-\mathrm{BPO}$ at $\mathrm{C}-5$ position in aqueous solutions is a well-known process, we provide a detailed study of the chemical stability of $\mathrm{BP}$ determinants at $\mathrm{pH}$ conditions normally used in order to further understand this progress. Our findings indicate that the epimerization rate is influenced by the structure of the determinant, changing dramatically also the kinetics of the process. In vitro immunoassays results show the importance of the spatial configuration of C-5 of benzylpenicilloyl determinants in the IgE recognition, with the original $(5 R, 6 R)$-configuration as the best recognized. Any change, albeit small, in the chemical structure of the antigenic determinant of BP significantly affects IgE recognition. Therefore, the inclusion of stereochemically pure compounds or mixtures may have important implications for the sensitivity of both in vivo and in vitro diagnostic tests.

The conclusions drawn for the BP determinants in this study could serve as basis for the evaluation of the determinants derived from the rest of penicillins. In addition, the conditions of $\mathrm{pH}$ and temperature in which these reagents can be handled, for avoiding degradation or epimerization, is crucial to properly use standardized reagents that lead to reproducible results.

\section{DATA AVAILABILITY STATEMENT}

The raw data supporting the conclusion of this article will be made available by the authors, without undue reservation, to any qualified researcher.

\section{ETHICS STATEMENT}

The studies involving human participants were reviewed and approved by the institutional review board, and informed consent for all procedures was obtained from all patients (Regional University Hospital of Málaga). The patients/participants provided their written informed consent to participate in this study.

\section{REFERENCES}

Antunez, C., Fernandez, T., Blanca-Lopez, N., Torres, M. J., Mayorga, C., Canto, G., et al. (2006). IgE antibodies to betalactams: relationship between the triggering hapten and the specificity of the immune response. Allergy 61 (8), 940-946. doi:10.1111/j.1398-9995.2006.01120.x

Ariza, A., Mayorga, C., Fernández, T. D., Barbero, N., Martín-Serrano, A., Pérez-Sala, D., et al. (2015). Hypersensitivity reactions to $\beta$-lactams: relevance of hapten-protein conjugates. J Investig. Allergol. Clin. Immunol. 25 (1), 12-25.

Ariza, A., Mayorga, C., Salas, M., Doña, I., Martín-Serrano, Á., Pérez-Inestrosa, E., et al. (2016). The influence of the carrier molecule on amoxicillin

\section{AUTHOR CONTRIBUTIONS}

EP-I, MIM, and YV conceived and designed the experiments; MIM performed the NMR experiments; FN performed DFT calculations; CM performed in vitro tests and analyzed RAST data; TDF made statistical analysis of the results of Competitive Inhibition immunoassay; GB and MJT evaluated and selected patients and controls; YV and MIM analyzed all the data and wrote the paper with input from CM and EP-I.

\section{FUNDING}

This work was supported by the Spanish Ministerio de Economía, Industria y Competitividad (CTQ2016-75870-P), Ministerio de Ciencia y Educación (PID2019-104293GB-I00), Ministerio de Ciencia e Innovación (Proyectos de $\mathrm{I}+\mathrm{D}+\mathrm{I}$ " Programación Conjunta Internacional», EuroNanoMed 2019 (PCI2019-111825-2), Instituto de Salud Carlos III (ISCIII) of MINECO (grants cofunded by ERDF: "Una manera de hacer Europa" (PI17/01237, PI18/00095, RETIC ARADYAL, RD16/0006/0001and RD16/0006/0012, Euronanomed Program AC19/00082, “Joan Rodés" program (JR18/00054) and Miguel Servet I program (CP15/00103), Junta de Andalucía and Universidad de Málaga (UMA18-FEDERJA-007), Andalusian Regional Ministry of Health (PI-0179-2014, PE-01722018) and Nicolas Monardes Program (RC-0004-2016C) and "Premio UNICAJA a la innovación en biomedicina y salud".

\section{ACKNOWLEDGMENTS}

We are indebted to the SCBI (Supercomputing and Bioinformatics Center) of the University of Malaga for providing computer resources, technical expertise, and assistance during this work. We thank Ms Claudia Corazza for her help with the English version of the manuscript.

\section{SUPPLEMENTARY MATERIAL}

The Supplementary Material for this article can be found online at: https://www.frontiersin.org/articles/10.3389/fphar.2021.585890/ full\#supplementary-material.

recognition by specific IgE in patients with immediate hypersensitivity reactions to betalactams. Sci. Rep. 6 (1), 35113-35210. doi:10.1038/srep35113

Bader, R. F. W. (1991). A quantum theory of molecular structure and its applications. Chem. Rev. 91 (5), 893-928. doi:10.1021/cr00005a013

Baldwin, J. E. (1976). Rules for ring closure. J. Chem. Soc., Chem. Commun. 18, 734-736. doi:10.1039/C39760000734

Batchelor, F. R., Dewdney, J. M., and Gazzard, D. (1965). Penicillin allergy: the Formation of the penicilloyl determinant. Nature 206 (4982), 362-364. doi:10. 1038/206362a0

Blanca, M., Mayorga, C., Perez, E., Suau, R., Juarez, C., Vega, J. M., et al. (1992). Determination of IgE antibodies to the benzyl penicilloyl determinant. A comparison between poly-l-lysine and human serum albumin as carriers. J. Immunol. Methods 153 (1-2), 99-105. doi:10.1016/0022-1759(92)90311-g 
Branch, S. K., Casy, A. F., and Ominde, E. M. (1987). Application of $1 \mathrm{H}$ nuclear magnetic resonance spectroscopy to the analysis of beta-lactam antibiotics and their common degradation products. J. Pharmaceut. Biomed. Anal. 5, 73. doi:10. 1016/0731-7085(87)80011-0

Brockow, K., Romano, A., Blanca, M., Ring, J., Pichler, W., and Demoly, P. (2002). General considerations for skin test procedures in the diagnosis of drug hypersensitivity. Allergy 57 (1), 45-51. doi:10.1046/j.0105-4538.2001.00001. $\mathrm{x}-\mathrm{i} 8$

Busson, R., and Vanderhaeghe, H. (1976). Preparation and stereochemical analysis of 5-epibenzylpenicillin (S)- and (R)-sulfoxide esters. J. Org. Chem. 41 (18), 3054-3056. doi:10.1021/jo00880a039

Davis, A. M., Jones, M., and Page, M. I. (1991). Thiazolidine ring opening in penicillin derivatives. Part 1. Imine formation. Journal of the Chemical Society, Perkin Transactions 2 (8), 1219-1223. doi:10.1039/P29910001219

Davis, A. M., and Page., M. I. (1985). Opening of the thiazolidine ring of penicillin derivatives. J. Chem. Soc., Chem. Commun. 23 (January), 1702-1704. doi:10. 1039/c39850001702

Demoly, P., Adkinson, N. F., Brockow, K., Castells, M., Chiriac, A. M., Greenberger, P. A., et al. (2014). International consensus on drug allergy. Allergy 69 (4), 420-437. doi:10.1111/all.12350

Doña, I., Romano, A., and Torres, M. J. (2019). Algorithm for betalactam allergy diagnosis. Allergy 74 (9), 1817-1819. doi:10.1111/all.13844

Doña, I., Torres, M. J., Montañez, M. I., and Fernández, T. D. (2017). In Vitro diagnostic testing for antibiotic allergy. Allergy, Asthma and Immunology Research 9 (4), 288-298. doi:10.4168/aair.2017.9.4.288

Fernández, J., Torres, M. J., Campos, J., Arribas-Poves, F., Blanca, M., and on behalf of the Dap-Diater Group (2013). Prospective, multicenter clinical trial to validate new products for skin tests in the diagnosis of allergy to penicillin. J. Invest. Allergol. Clin. Immunol. 23 (6), 398-408. Available at: http://www.jiaci. org/summary/vol23-issue6-num1052.

Fernandez, T. D., Mayorga, C., Salas, M., Barrionuevo, E., Posadas, T., Ariza, A., et al. (2017). Evolution of diagnostic approaches in betalactam hypersensitivity. Expet Rev. Clin. Pharmacol. 10, 671. doi:10.1080/ 17512433.2017.1313110

Fontaine, C., Mayorga, C., Bousquet, P. J., Arnoux, B., Torres, M. J., Blanca, M., et al. (2007). Relevance of the determination of serum-specific IgE antibodies in the diagnosis of immediate beta-lactam allergy. Allergy 62 (1), 47-52. doi:10. $1111 /$ j.1398-9995.2006.01268.x

Frisch, M. J., Trucks, G. W., Schlegel, H. B., Scuseria, G. E., Robb, M. A., Cheeseman, J. R., et al. (2016). Gaussian 09, revision A.02. Wallingford, CT: Gaussian, Inc. Available at: http://gaussian.com/g09citation/.

Ghebre-Sellassie, I., Knevel, A. M., and Hem, S. L. (1984). Epimerization of benzylpenicilloic acid in alkaline media. J. Pharmaceut. Sci. 73 (1), 125-128. doi:10.1002/jps.2600730135

Haginaka, J., and Wakai, J. (1985). Epimerization of benzylpenicilloate in alkaline aqueous solutions. Chem. Pharm. Bull. 33 (6), 2605-2608. doi:10.1248/cpb.33. 2605

Johansson, S. G. O., Adédoyin, J., Van Hage, M., Grönneberg, R., and Nopp, A. (2013). . False-positive penicillin immunoassay: an unnoticed common problem. J. Allergy Clin. Immunol. 132 (1), 235-237. doi:10.1016/j.jaci.2012. 11.017

Joint Task Force on Practice ParametersAmerican Academy of AllergyAsthma and ImmunologyAmerican College of AllergyAsthma and ImmunologyJoint Council of Allergy, et al. (2010). Drug allergy: an updated Practice parameter. Ann. Allergy Asthma Immunol. 105 (4), 259-273. doi:10.1016/j. anai.2010.08.002

Lacombe-Barrios, J., Salas, M., Gómez, F., Doña, I., Ariza, A., Mayorga, C., et al. (2016). The addition of benzylpenicillin does not increase the skin test sensitivity obtained with classic $\beta$-lactam determinants. J Investig. Allergol. Clin. Immunol. 26, 52. doi:10.18176/jiaci.0009

Levine, B. B., and Ovary, Z. (1961). Studies on the mechanism of the formation of the penicillin antigen. III. The N-(D-alpha-benzylpenicilloyl) group as an antigenic determinant responsible for hypersensitivity to penicillin G. J. Exp. Med. 114 (6), 875-904. doi:10.1084/jem.114.6.875

Llinás, A., Vilanova, B., Muñoz, F., and Donoso, J. (2001). The role of a $\beta$-proton transfer donor in the degradation of benzylpenicillin. J. Mol. Catal. Chem. 175 (1-2), 3-16. doi:10.1016/S1381-1169(01)00203-5
Lu, T., and Chen, F. (2012). Multiwfn: a multifunctional wavefunction analyzer. J. Comput. Chem. 33 (5), 580-592. doi:10.1002/jcc.22885

Lu, T. (2020). Multiwfn manual, version 3.6(dev), section 3.14Multiwfn manual, version 3.6(dev). Available at: http://sobereva.com/multiwfn/.

Macy, E., Goldberg, B., and Poon, K. Y. (2010). Use of commercial anti-penicillin IgE fluorometric enzyme immunoassays to diagnose penicillin allergy. Ann. Allergy Asthma Immunol. 105 (2), 136-141. doi:10.1016/j.anai.2010.06.014

Martin-Serrano, A., Barbero, N., Agundez, J. A., Vida, Y., Perez-Inestrosa, E., and Montanez, M. I. (2016). New advances in the study of IgE drug recognition. Curr. Pharmaceut. Des. 22 (45), 6759-6772. doi:10.2174/ 1381612822666160921142231

Martín-Serrano, A., Mayorga, C., Barrionuevo, E., Pérez, N., Romano, A., Moreno, E., et al. (2020). Design of an antigenic determinant of cefaclor: chemical structure-IgE recognition relationship. J. Allergy Clin. Immunol. 145 (4), 1301-1304. doi:10.1016/j.jaci.2019.11.036

Mayorga, C., Celik, G., Rouzaire, P., Whitaker, P., Bonadonna, P., RodriguesCernadas, J., et al. (2016a). In Vitro tests for drug hypersensitivity reactions: an ENDA/EAACI drug allergy interest group position paper. Allergy 71 (8), 1103-1134. doi:10.1111/all.12886

Mayorga, C., Perez-Inestrosa, E., Molina, N., Montañez, M. I., and Montañez (2016b). Development of nanostructures in the diagnosis of drug hypersensitivity reactions. Curr. Opin. Allergy Clin. Immunol. 16 (4), 300-307. doi:10.1097/ACI.0000000000000282

Montañez, M. I., Ariza, A., Mayorga, C., Diana Fernandez, T., and Torres, M. J. (2015). Cross-reactivity in betalactam allergy: alternative treatments. Current Treatment Options in Allergy 2 (2), 141-154. doi:10.1007/s40521015-0050-4

Montañez, M. I., Mayorga, C., Torres, M. J. M. B., and Perez-Inestrosa, E. (2011a). Methodologies to anchor dendrimeric nanoconjugates to solid phase: toward an efficient in Vitro detection of allergy to $\beta$-lactam antibiotics. Nanomed. Nanotechnol. Biol. Med. 7 (6), 682-685. doi:10. 1016/j.nano.2011.07.008

Montañez, M. I., Perez-Inestrosa, E., Suau, R., Mayorga, C., Torres, M. J., and Blanca, M. (2008). Dendrimerized cellulose as a scaffold for artificial antigens with applications in drug allergy diagnosis. Biomacromolecules 9 (5), 1461-1466. doi:10.1021/bm701380a

Montañez, M. I., Najera, F., and Perez-Inestrosa, E. (2011b). NMR studies and molecular dynamic simulation of synthetic dendritic antigens. Polymers 3 (3), 1533-1553. doi:10.3390/polym 3031533

Munro, A. C., Chainey, M. G., and Woroniecki, S. R. (1978). Preparation and immunological cross-reactions of penicilloic and penilloic acids. J. Pharmacol. Sci. 67 (9), 1197-1204. doi:10.1002/jps.2600670903

Neftel, K. A., Wälti, M., Schulthess, H. K., and Gubler, J. (1984). Adverse reactions following intravenous penicillin-G relate to degradation of the drug in Vitro. Klin. Wochenschr. 62 (1), 25-29. doi:10.1007/BF01725189

Neftel, K. A., Wälti, M., Spengler, H., and De Weck, A. L. (1982). Effect of storage of penicillin-G solutions on sensitisation to penicillin-G after intravenous administration. Lancet 1 (8279), 986-988. doi:10.1016/S0140-6736(82)91991-2

Pajares, M. A., Zimmerman, T., Sánchez-Gómez, F. J., Sánchez-Gómez, F. J. A., Ariza, A., Torres, M. J., et al. (2020). Amoxicillin inactivation by thiol-catalyzed cyclization reduces protein haptenation and antibacterial potency. Front. Pharmacol. 11, 189. doi:10.3389/fphar.2020.00189

Romano, A., Atanaskovic-Markovic, M., Barbaud, A., Bircher, A. J., Brockow, K., Caubet, J. C., et al. (2020). Towards a more precise diagnosis of hypersensitivity to beta-lactams - an EAACI position paper. Allergy 75 (6), 1300-1315. doi:10. 1111/all.14122

Ruiz-Sanchez, A. J., Montañez, M. I., Mayorga, C., Torres, M. J., Kehr, N. S., Vida, Y., et al. (2012). Dendrimer-modified solid supports: nanostructured materials with potential drug allergy diagnostic applications. Curr. Med. Chem. 19 (29), 4942-4954. doi:10.2174/0929867311209024942

Sánchez-Sancho, F., Pérez-Inestrosa, E., Suau, R., Mayorga, C., Torres, M. J., and Blanca, M. (2002). Dendrimers as carrier protein mimetics for IgE antibody recognition. Synthesis and characterization of densely penicilloylated dendrimers. Bioconjugate Chem. 13 (3), 647-653. doi:10. $1021 / \mathrm{bc} 0155824$

Tomasi, J., Mennucci, B., and Cammi, R. (2005). Quantum mechanical continuum solvation models. Chem. Rev., 105, 2999. doi:10.1021/cr9904009 
Torres, M. J., Ariza, A., Fernández, J., Moreno, E., Laguna, J. J., Montañez, M. I., et al. (2010). Role of minor determinants of amoxicillin in the diagnosis of immediate allergic reactions to amoxicillin. Allergy 65 (5), 590-596. doi:10. 1111/j.1398-9995.2009.02245.x

Torres, M. J., Blanca, M., Fernandez, J., Romano, A., Weck, A., Aberer, W., et al. (2003). Diagnosis of immediate allergic reactions to beta-lactam antibiotics. Allergy 58 (10), 961-972. doi:10.1034/j.1398-9995.2003.00280.x

Torres, M. J., Romano, A., Celik, G., Demoly, P., Khan, D. A., Macy, E., et al. (2017). Approach to the diagnosis of drug hypersensitivity reactions: similarities and differences between Europe and North America. Clin. Transl. Allergy 7, 7. doi:10.1186/s13601-017-0144-0

Vida, Y., Montañez, M. I., Collado, D., Najera, F., Ariza, A., Blanca, M., et al. (2013). Dendrimeric antigen-silica particle composites: an innovative approach for IgE quantification. J. Mater. Chem. B 1 (24), 3044-3050. doi:10.1039/c3tb20548g

Conflict of Interest: The authors collaborate in research grants with Diater Laboratories (Madrid, Spain).

Copyright (๐ 2021 Mayorga, Montañez, Najera, Bogas, Fernandez, Gil, Palacios, Torres, Vida, Perez-Inestrosa. This is an open-access article distributed under the terms of the Creative Commons Attribution License (CC BY). The use, distribution or reproduction in other forums is permitted, provided the original author(s) and the copyright owner(s) are credited and that the original publication in this journal is cited, in accordance with accepted academic practice. No use, distribution or reproduction is permitted which does not comply with these terms. 